\title{
Thromboembolism after pneumonectomy for malignancy: An independent marker of poor outcome
}

David P. Mason, MD, ${ }^{a}$ Mohammad A. Quader, MD, ${ }^{a}$ Eugene H. Blackstone, MD, ${ }^{a}, b$ Jeevanantham Rajeswaran, MSc,

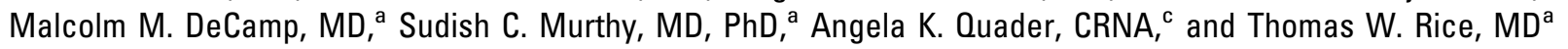

From the Departments of Thoracic and Cardiovascular Surgery, ${ }^{\text {a }}$ Quantitative Health Sciences, ${ }^{\mathrm{b}}$ and General Anesthesiology, ${ }^{\mathrm{c}}$ The Cleveland Clinic Foundation, Cleveland, Ohio.

Received for publication July 29, 2005; revisions received Oct 11, 2005; accepted for publication Oct 26, 2005.

Address for reprints: David P. Mason, MD, Department of Thoracic and Cardiovascular Surgery, The Cleveland Clinic Foundation, 9500 Euclid Ave/Desk F24, (E-mail: masond2@ccf.org).

J Thorac Cardiovasc Surg 2006;131:711-8

$0022-5223 / \$ 32.00$

Copyright $@ 2006$ by The American Association for Thoracic Surgery

doi:10.1016/j.jtcvs.2005.10.027
Objective: Because venous thromboembolism results in important postoperative morbidity and mortality after pneumonectomy for malignancy, we sought to determine its prevalence, location, management, timing, and risk factors. We also evaluated short- and long-term outcomes of patients in whom venous thromboembolism developed compared with those of patients in whom it did not.

Methods: Between January 1990 and January 2001, 336 patients underwent pneumonectomy for malignancy. Patients were considered to have venous thromboembolism if they were identified as having deep vein thrombosis or pulmonary embolus through chart review, including pulmonary imaging studies. All patients were managed with anticoagulation or anticoagulation plus thrombolysis.

Results: Twenty-five (7.4\%) patients had postoperative venous thromboembolism, with peak incidence 7 days after the operation; most had already been discharged from the hospital. Higher pack-years of smoking was associated with increased risk, as well as with earlier occurrence of venous thromboembolism $(P<.04)$. Survival was $55 \%$ at 6 months and $13 \%$ at 18 months; mode of death was cancer in $14(61 \%)$ of 23 , respiratory failure in $4(17 \%)$ of 23 , multisystem organ failure in $3(13 \%)$ of 23 , myocardial infarction in $1(4.4 \%)$ of 23 , and uncertain in $1(4.4 \%)$ of 23 . Low preoperative forced vital capacity was predictive of poor long-term survival $(P=$ $.02)$. Patients with venous thromboembolism had substantially lower survival than predicted from competing-risks analysis of survival without venous thromboembolism (13\% vs $60 \%$ at 18 months), and this difference persisted after censoring for deaths directly attributable to venous thromboembolism.

Conclusions: Venous thromboembolism is surprisingly common after pneumonectomy for malignancy and portends poor survival. Improved screening and better prophylaxis might prevent this complication and enhance outcome.

$\mathrm{D}$ eep vein thrombosis complicates the postoperative course of patients undergoing surgical intervention for cancer more often than that of patients undergoing surgical intervention for benign disease. ${ }^{1}$ This is particularly true for patients with lung cancer, whose risk of venous thromboembolism (VTE) is 20 times greater than that of the general population, with an incidence of 44.4 per 1000 person-years. ${ }^{2}$ Patients who undergo thoracotomy and pulmonary resection for cancer are at high risk for VTE, perhaps because in addition to the thromboembolic risk of a major operation, they manifest a hypercoaguable state caused by their underlying malignancy. Because of compromised pulmonary reserve, patients undergoing pneumonectomy have the most at stake from thromboembolism and represent the highest surgical risk.

Therefore, we sought to determine the prevalence, location, management, timing, and risk factors for VTE after pneumonectomy for cancer. We also evaluated short- 


\section{Abbreviations and Acronyms}

$\mathrm{VTE}=$ venous thromboembolism

and long-term outcomes of patients in whom VTE developed compared with those of patients in whom it did not.

\section{Patients and Methods Patients}

From January 1990 through January 2001, 336 patients underwent pneumonectomy for cancer at The Cleveland Clinic Foundation. Data were extracted from the Thoracic Surgery Registry, which has been approved for research by the institutional review board, and supplemented by detailed review of all medical records.

\section{Prophylaxis and Diagnosis of VTE}

Postoperative prophylaxis for VTE was subcutaneous heparin, 5000 units twice daily, and pneumatic stockings through hospital discharge. All patients were ambulated immediately postoperatively, as well as after VTE, unless cardiorespiratory compromise prohibited it.

Clinical signs of VTE were observed on history and physical examination and included upper or lower extremity swelling, calf pain, and shortness of breath. Clinical findings were confirmed by imaging for deep vein thrombosis, pulmonary embolism, or both. Deep vein thrombosis was confirmed by means of Doppler ultrasonography; occasionally, venography was performed for diagnosis and therapy. No preoperative screening for deep vein thrombosis was performed, and all vases of VTE were diagnosed from clinical suspicion. Patients were instructed to call after discharge to report symptoms of upper or lower extremity swelling, calf pain, or shortness of breath and then were readmitted.

Deep vein thrombosis of the upper extremity was defined as involving the basilic, axillary, brachiocephalic, and internal jugular veins. Lower extremity deep vein thrombosis included the common femoral, superficial femoral, popliteal, tibial, and soleal veins. Central venous thrombosis included the superior or inferior vena cava, as well as the iliac veins. Standard criteria for noncompressible echogenic filling defects defined by our Vascular Medicine Laboratory were used to identify deep vein thrombosis. Venography was not required for confirmation. Pulmonary embolus was diagnosed on the basis of a mismatched defect on ventilationperfusion scanning coupled with clinical suspicion. Ventilationperfusion scans and pulmonary angiograms were largely abandoned with the advent of pulmonary embolism protocol computed tomography.

\section{Outcomes}

Primary outcome was all-cause mortality. Vital status was obtained by means of systematic follow-up augmented by the Social Security Death Index. ${ }^{3,4}$ Median follow-up was 1.6 years, with 828 patient-years available for analysis. When possible, we obtained mode of death from follow-up contact with families and autopsy and chart review.

\section{Timing of VTE and its Risk Factors}

Timing of VTE was determined from the date of the operation to the date of diagnosis. Interval from VTE to hospital discharge was also recorded. Estimates of freedom from occurrence of VTE were obtained by using the Kaplan-Meier method and by using a parametric method that resolves the number of phases of instantaneous risk and estimates shaping parameters. ${ }^{5}$ This method allowed us to determine the time of peak identification of VTE, as well as risk of death.

Risk factors for VTE were identified in the hazard function domain (see Appendix 1 for list of variables included in the analysis). ${ }^{5}$ Continuous and ordinal variables were retained in their original state to maximize information content, with original measurement scales calibrated to assumptions of the analysis by transformation as necessary (linearization). Variable selection used bootstrap aggregation (bagging), with automated analyses of 1000 random data sets performed by using a variable retention criterion of a $P$ value of less than $.1 .^{6,7}$ Resulting models were aggregated, and results wee expressed as the frequency of occurrence of both single factors and closely related clusters of factors. We included any factor appearing in at least $50 \%$ of the analyses. In addition, we included the most frequently occurring representative for any cluster appearing in at least $50 \%$ of the analyses.

Coefficients in multivariable models are presented with 1 standard error of the estimates, and freedom from events and hazard estimates are accompanied by asymmetric $68 \%$ confidence limits equivalent to 1 standard error. Hazard ratios were not used because the model does not assume proportional hazards and because transformations were made of continuous variables that made hazard ratios uninterpretable.

\section{Comparison of Outcome}

To determine the effect of VTE on outcome, we used competingrisks analysis to compare survival of patients before VTE developed with that after VTE was diagnosed. ${ }^{8}$ Competing events were (1) death before VTE and (2) VTE. Risk factors for death before VTE were identified as described above. Then for each patient experiencing VTE, a survival curve was predicted as if he or she had not experienced VTE (expected survival). These curves were aggregated and compared with observed survival. ${ }^{9}$

\section{Results}

\section{Prevalence, Location, and Management of VTE}

Prevalence of VTE was $7.4 \%(25 / 336) ; 17$ (68\%) patients had deep vein thrombosis only, $5(20 \%)$ had pulmonary embolism only, and $3(12 \%)$ had both. The 20 cases of confirmed deep vein thrombosis occurred in 33 locations, with some patients having more than one focus of thrombosis. These included 14 upper extremity, 15 lower extremity, and 4 central (iliac and vena cava) cases.

All patients were immediately anticoagulated with intravenous heparin and later converted to warfarin; 2 with hemodynamic instability and pulmonary embolism underwent thrombolysis. Of the 8 patients with pulmonary embolism admitted to the intensive care unit for respiratory compromise, 7 required intubation, and 1 had inferior vena cava filter placement. One patient with deep vein thrombo- 


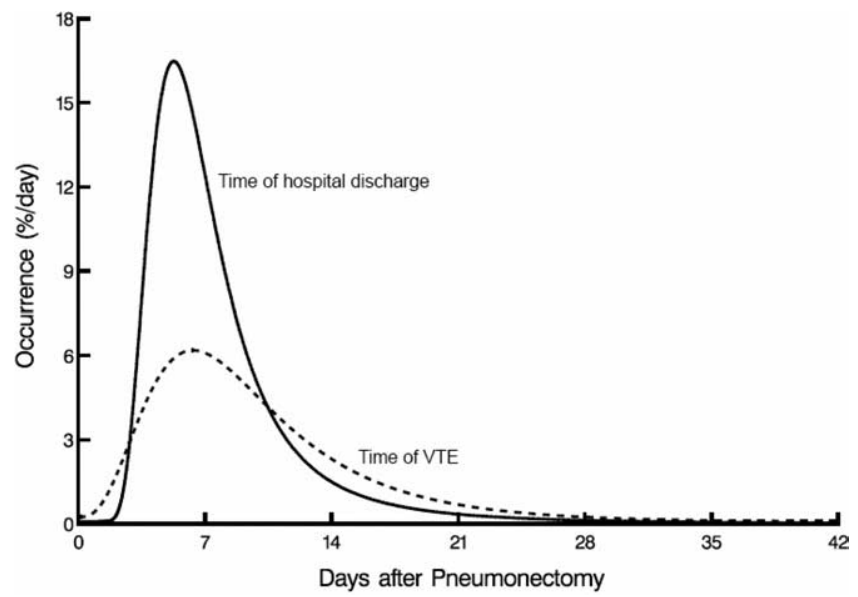

Figure 1. Timing of venous thromboembolism (VTE) relative to time of hospital discharge after pneumonectomy.

sis alone had inferior vena cava filter placement. No patient with VTE had heparin-induced thrombocytopenia, and no patient had major bleeding requiring reoperation after anticoagulation.

Six patients were given diagnoses of VTE preoperatively; 4 of these had deep vein thrombosis and were treated perioperatively with standard prophylaxis, including subcutaneous heparin. One patient with preoperative deep vein thrombosis had an acute deep vein thrombosis postoperatively. Two patients had pulmonary embolism preoperatively; 1 was treated with intravenous heparin perioperatively and the other with an inferior vena cava filter. Neither experienced postoperative pulmonary embolism.

\section{Timing of VTE and Its Risk Factors}

The incidence of VTE peaked 7 days after the operation, and most patients (64\%) who had diagnoses of VTE had already been discharged; only $9(36 \%)$ had it while still in the hospital (Figure 1). Postoperative length of stay of patients undergoing pneumonectomy decreased during this study, from approximately 14 days in 1990 to 7 days in 2000. This trend made it increasingly likely that patients would have VTE at home and require readmission. The incidence of VTE after the first 2 postoperative weeks decreased rapidly, and few events occurred after the first month (Figure 2).

Higher pack-years of smoking was identified as a preoperative risk factor for VTE (Table 1). The more pack-years patients smoked, the higher their likelihood of having VTE (Figure 3).

\section{Death After VTE and Its Risk Factors}

Survival after VTE diagnosis was 55\%, 34\%, and $13 \%$ at 6, 12 , and 18 months, respectively. We attempted to determine

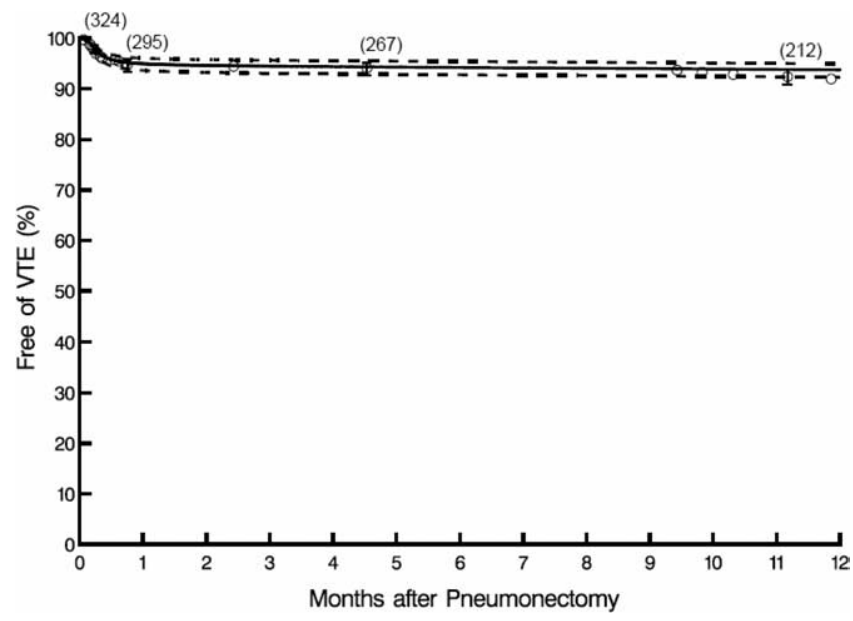

Figure 2. Freedom from venous thromboembolism (VTE) after pneumonectomy. Each circle represents an event, vertical bars are $68 \%$ confidence limits equivalent to 1 standard error, and numbers in parentheses are numbers of patients remaining at risk. The solid line enclosed within dashed $68 \%$ confidence limits is the parametric estimate that generated the hazard function shown in Figure 1.

whether patients with pulmonary embolism had worse survival than those with deep vein thrombosis and found no difference $(P=.7)$. Furthermore, we found no difference in outcomes in patients with upper versus lower limb deep vein thrombosis. Pulmonary embolism occurred more often in patients with lower extremity (2/9) than upper extremity deep vein thrombosis (1/8), but the difference was not statistically significant.

The only risk factor for death after VTE was lower forced vital capacity expressed as a percentage of normal value $(P=.02)$. This was particularly true for patients with a forced vital capacity of less than $80 \%$, who experienced a steep decrease in 1-year survival (Figure 4).

Mode of death was cancer in $14(61 \%)$, respiratory failure in $4(17 \%)$, multisystem organ failure in $3(13 \%)$, myocardial infarction in $1(4.4 \%)$, and uncertain in 1 (4.4\%).

\section{Comparison of Outcome}

Was mortality higher than expected in patients with VTE? Expected survival was calculated on the basis of the competing risk of death before VTE. Risk factors for death included male sex, preoperative radiotherapy, lower forced expiratory volume in 1 second (percentage of normal value), higher pT, and increased pack-years of smoking (Table 2). Expected survival was considerably higher than observed survival (60\% vs $13 \%$ at 18 months; Figure 5). This difference persisted after censoring 3 deaths directly attributable to $\operatorname{VTE}(60 \%$ vs $15 \%)$. 
TABLE 1. Preoperative patient and tumor characteristics

\begin{tabular}{|c|c|c|c|c|}
\hline \multirow[b]{2}{*}{ Characteristic } & \multirow[b]{2}{*}{ Overall $(\mathbf{n}=336)$, no. $(\%)$} & \multicolumn{2}{|c|}{ Venous thromboembolism } & \multirow[b]{2}{*}{$P$ value } \\
\hline & & Yes $(n=25)$, no. $(\%)$ & No $(\mathrm{n}=311)$, no. $(\%)$ & \\
\hline \multicolumn{5}{|l|}{ Patient } \\
\hline Male sex & $248(74)$ & $19(76)$ & $229(74)$ & .8 \\
\hline Age $(y)^{*}$ & $61 \pm 11$ & $61 \pm 13$ & $61 \pm 11$ & .9 \\
\hline BMI $\left(\mathrm{kg} \cdot \mathrm{m}^{-2}\right)^{*}$ & $26 \pm 5$ & $26 \pm 5$ & $26 \pm 5$ & .7 \\
\hline \multirow{2}{*}{\multicolumn{5}{|c|}{$\begin{array}{l}\text { Pulmonary function tests } \\
\mathrm{FEV}_{1}{ }^{*}\end{array}$}} \\
\hline & & & & \\
\hline Liters & $2.4 \pm 0.65$ & $2.3 \pm 0.45$ & $2.4 \pm 0.66$ & .5 \\
\hline$\%$ of normal value & $73 \pm 18$ & $72 \pm 12$ & $72 \pm 19$ & .8 \\
\hline \multicolumn{5}{|l|}{$\mathrm{FVC}^{*}$} \\
\hline Liters & $3.5 \pm 0.87$ & $3.3 \pm 0.74$ & $3.5 \pm 0.90$ & .4 \\
\hline$\%$ of normal value & $85 \pm 17$ & $82 \pm 13$ & $84 \pm 20$ & .5 \\
\hline $\mathrm{FVC} / \mathrm{FEV}_{1}$ & $0.69 \pm 0.11$ & $0.71 \pm 0.11$ & $0.68 \pm 0.12$ & .3 \\
\hline Smoker (pack-years)† & $24,45,80$ & $30,50,100$ & $22,45,80$ & .02 \\
\hline \multicolumn{5}{|l|}{ Comorbidity } \\
\hline Insulin-treated diabetes $\ddagger$ & $7(2)$ & $0(0)$ & $7(2)$ & 1.0 \\
\hline Non-insulin-treated diabetes & $25(7)$ & $2(8)$ & $23(7)$ & 1.0 \\
\hline History of hypertension & $113(34)$ & $7(28)$ & $106(34)$ & .5 \\
\hline Coronary artery disease $§$ & $63(19)$ & $4(16)$ & $59(19)$ & 1.0 \\
\hline History of AF $\ddagger$ & $18(5)$ & $1(4)$ & $17(6)$ & 1.0 \\
\hline History of DVT & $4(1)$ & $1(4)$ & $3(1)$ & .3 \\
\hline History of PE & $2(0.6)$ & $0(0)$ & $2(0.6)$ & 1.0 \\
\hline History of CVA & $16(5)$ & $0(0)$ & $16(5)$ & .6 \\
\hline \multicolumn{5}{|l|}{ Primary lung cancer } \\
\hline Histology\| & & & & .8 \\
\hline Squamous cell & $119(36)$ & $11(52)$ & $108(35)$ & \\
\hline Adenocarcinoma & $186(56)$ & $9(43)$ & $177(57)$ & \\
\hline Mixed type & $2(0.6)$ & $0(0)$ & $2(0.6)$ & \\
\hline Mesothelioma & $14(4)$ & $1(5)$ & $13(4)$ & \\
\hline Small cell & $2(0.6)$ & $0(0)$ & $2(0.6)$ & \\
\hline Metastatic & $7(12)$ & $0(0)$ & $7(2)$ & \\
\hline Renal & $1(0.6)$ & $0(0)$ & $1(0.3)$ & \\
\hline Unknown & $1(0.3)$ & $0(0)$ & $1(0.3)$ & \\
\hline pT classification? & & & & .3 \\
\hline 1 & $51(16)$ & $1(5)$ & $50(17)$ & \\
\hline 2 & $168(54)$ & $14(74)$ & $154(52)$ & \\
\hline 3 & $57(18)$ & $3(16)$ & $54(18)$ & \\
\hline 4 & 37 (12) & $1(5)$ & $36(12)$ & \\
\hline pN classification? & & & & $>.9$ \\
\hline 0 & $100(32)$ & $6(32)$ & $94(32)$ & \\
\hline 1 & $96(31)$ & $6(32)$ & $90(31)$ & \\
\hline 2 & 107 (34) & $6(32)$ & $101(34)$ & \\
\hline 3 & $10(3)$ & $1(5)$ & $9(3)$ & \\
\hline pM classification? & & & & 1.0 \\
\hline 0 & 303 (97) & $19(100)$ & $284(97)$ & \\
\hline 1 & $10(3)$ & $0(0)$ & $10(3)$ & \\
\hline Stage\# & & & & .6 \\
\hline IA & $21(7)$ & $0(0)$ & $21(7)$ & \\
\hline IB & $52(17)$ & $4(21)$ & $48(16)$ & \\
\hline$\| \mathrm{A}$ & $11(4)$ & $0(0)$ & $11(4)$ & \\
\hline IIB & $72(23)$ & $7(37)$ & $65(22)$ & \\
\hline IIIA & 105 (34) & $6(32)$ & $99(34)$ & \\
\hline IIIB & 41 (13) & $2(10)$ & $39(13)$ & \\
\hline IV & $10(3)$ & $0(0)$ & $10(3)$ & \\
\hline
\end{tabular}


TABLE 1. Continued

\begin{tabular}{|c|c|c|c|c|}
\hline \multirow[b]{2}{*}{ Characteristic } & \multirow[b]{2}{*}{ Overall $(n=336)$, no. $(\%)$} & \multicolumn{2}{|c|}{ Venous thromboembolism } & \multirow[b]{2}{*}{$P$ value } \\
\hline & & Yes $(n=25)$, no. $(\%)$ & No $(n=311)$, no. $(\%)$ & \\
\hline \multicolumn{5}{|l|}{ Preoperative therapy ${ }^{* *}$} \\
\hline Chemotherapy & $68(21)$ & $4(19)$ & $64(21)$ & 1.0 \\
\hline Radiotherapy & $69(21)$ & $5(24)$ & $64(21)$ & .8 \\
\hline Chemoradiotherapy & $57(17)$ & $4(19)$ & $53(17)$ & .8 \\
\hline
\end{tabular}

$B M I$, Body mass index; $F E V$, forced expiratory volume in 1 second; $F V C$, forced vital capacity; $A F$, atrial fibrillation; $D V T$, deep vein thrombosis; $P E$, pulmonary embolism; CVA, cerebrovascular accident. *Mean \pm standard deviation. †Fifteenth, 50th, and 85th percentiles. $\ddagger$ Three missing in "No" group. §One missing in "No" group. "Four missing in "Yes" group. ISSix missing in "Yes" group, and 17 missing in "No" group. \#Six missing in "Yes" group, and 18 missing in "No" group. **Not mutually exclusive; 4 missing in "Yes" group, and 6 missing in "No" group.

\section{Discussion}

Patients with thoracic malignancies undergoing surgical lung resection are at particular risk for morbidity or mortality from VTE. Typically, they are smokers with some component of chronic obstructive pulmonary disease. Pulmonary embolism has been found to be an important cause of acute fatality after lung resection, with $1.85 \%$ of patients in one study dying of acute cardiorespiratory failure, the majority because of pulmonary embolus. ${ }^{10}$

Surgical resection reduces respiratory reserve and the ability to tolerate VTE. Patients undergoing pneumonectomy represent the highest risk category because their respiratory reserve is most compromised, and their increased pulmonary artery pressures and decreased pulmonary vascular bed leave little margin to tolerate VTE.

\section{Principal Findings}

Prevalence, location, and management of VTE. We found no difference in outcome between patients with deep vein thrombosis and those with pulmonary embolism. Up-

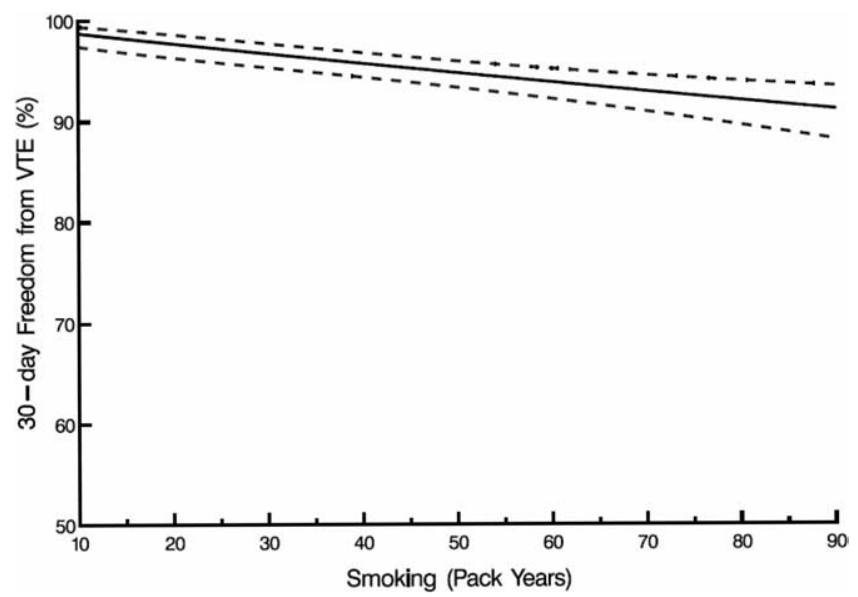

Figure 3. Freedom from venous thromboembolism (VTE) at $\mathbf{3 0}$ days after pneumonectomy for cancer according to pack-years of smoking (univariable analysis). The solid line is the point estimate enclosed within dashed $68 \%$ confidence limits. per extremity deep vein thromboses were surprisingly common, most likely related to central line placement. We routinely place central lines in all patients undergoing pneumonectomy, and all patients with upper extremity deep vein thrombosis in our study had central lines. This is consistent with studies showing that symptomatic catheter-related thrombosis occurs in $4 \%$ to $14 \%$ of patients with a central line, with the highest incidence in patients with cancer. ${ }^{11}$ Chemotherapeutic infusion or mediastinal radiation might add to the venous injury, contributing to deep vein thrombosis. ${ }^{12-14}$ Although there is a perception that deep vein thrombosis is less worrisome when it occurs in the upper extremities, we found that this complication clearly carries risk: 1 of 8 patients with upper extremity deep vein thrombosis also had pulmonary embolism, and long-term survival was decreased. We presently make an effort to minimize central line use and remove catheters early after surgical intervention.

Managing VTE after pneumonectomy is challenging. Mortality is high, particularly in the setting of pulmonary embolism, suggesting that management practices can be

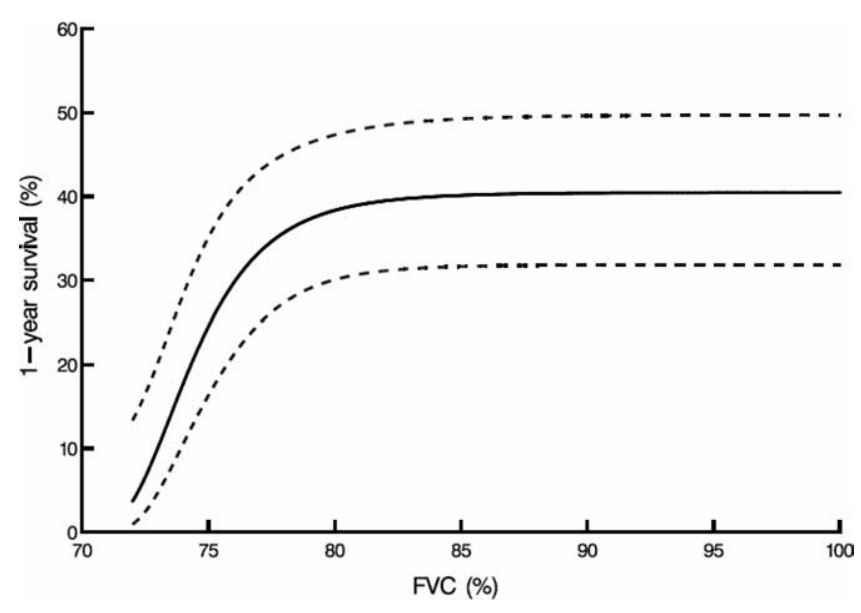

Figure 4. One-year survival after venous thromboembolism according to forced vital capacity (FVC) expressed as a percentage of normal value (univariable analysis). Depiction is as in Figure 3. 
TABLE 2. Incremental risk factors for death before venous thromboembolism

\begin{tabular}{lcc}
\hline Risk factor & Coefficient \pm SE & $P$ value \\
\hline Early hazard phase & & \\
$\quad$ None & & \\
Late hazard phase & & \\
$\quad$ Male sex & $0.43 \pm 0.17$ & .01 \\
Lower FEV $_{1}(\%$ of normal value) & \\
History of smoking & $0.98 \pm 0.44$ & .03 \\
Pack-years $\dagger$ & $1.1 \pm 0.79$ & .10 \\
Pack-years & $-5.9 \pm 2.2$ & .007 \\
Higher pT stage§ & $-1.1 \pm 0.45$ & .02 \\
Preoperative radiotherapy & $0.37 \pm 0.17$ & .03 \\
\end{tabular}

$S E$, Standard error; $F E V_{1}$, forced expiratory volume in 1 second. *(1/FEV $)^{2}$ inverse squared transformation. $\dagger(50 /$ smoking pack-years +1$)$, inverse transformation. $\ddagger$ Ln(smoking pack-years +1 ), logarithmic transformation. $\S \operatorname{Ln}(\mathrm{pT}$ stage), logarithmic classification.

improved. We instituted therapeutic intravenous anticoagulation with heparin in all patients but proceeded to thrombolysis in only 1 patient, who survived. We are reluctant to institute thrombolysis early postoperatively because it could lead to bleeding into the pneumonectomy space, although we did not have this complication. Given the overall high mortality, proceeding to thrombolysis earlier might be warranted. This has been shown to result in greater and faster improvement in angiographic and hemodynamic parameters when compared with heparin alone in patients with pulmonary embolism. ${ }^{15}$ Catheter-based or operative thrombectomy might also be considered as an early intervention.

Inferior vena cava filters were successfully used in 1 patient with pulmonary embolism preoperatively, as well as in 1 patient who had postoperative deep vein thrombosis. Although the majority of our patients with VTE had deep vein thrombosis alone, a substantial proportion had pulmonary embolism with important mortality. For this reason, we strongly consider inferior cava filter placement in all patients with VTE undergoing pneumonectomy, particularly those with proximal lower extremity deep vein thrombosis.

Timing of VTE and its risk factors. The fact that $7.4 \%$ of our patients had VTE postoperatively and more than half of these had it after being discharged suggests that our prophylaxis regimen needs to be improved and possibly lengthened. All patients received pneumatic compression stockings and 5000 units of unfractionated heparin twice a day. Nevertheless, the prevalence of deep vein thrombosis and pulmonary embolism was high, although lower than in the postthoracotomy patients studied by Ziomek and colleagues, ${ }^{16}$ who detected postoperative thromboembolism in $19 \%$ of patients. Our results were comparable with those of a large series of patients who underwent pneumonectomy for malignant mesothelioma. In that study deep vein throm-

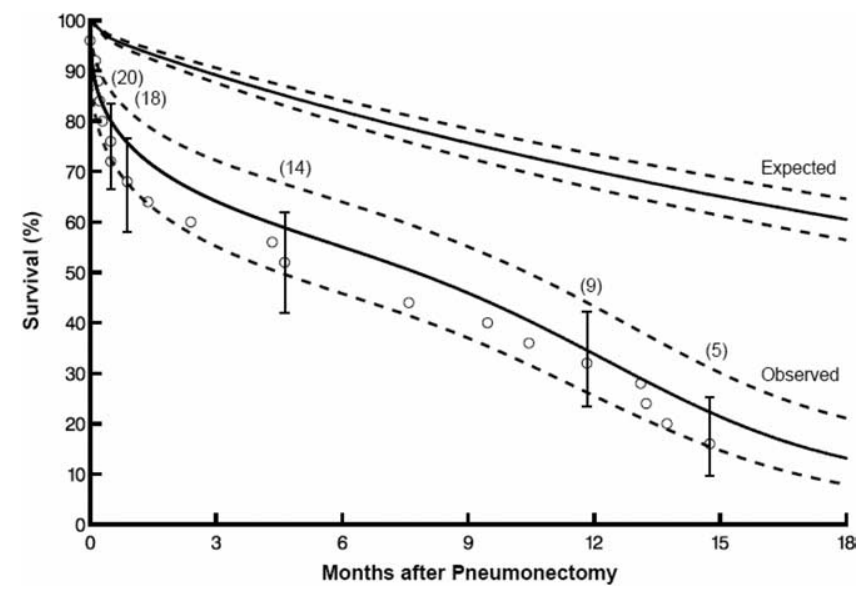

Figure 5. Expected upper solid curve enclosed in $68 \%$ confidence levels versus observed (circles) survival after diagnosis of venous thromboembolism. Actuarial and parametric depiction of the latter is as in Figure 2.

bosis occurred in $6.4 \%$ of patients postoperatively, and pulmonary embolism occurred in $1.5 \% .{ }^{17}$

Early hospital discharge after surgical intervention might be shifting occurrence of VTE to the outpatient setting, leading to underestimation of risk. ${ }^{18} \mathrm{We}$ are considering whether another form of prophylaxis should be used, such as low-molecular-weight heparin or routine addition of warfarin, as recommended for other high-risk patient populations. ${ }^{19,20}$ Low-molecular-weight heparin (enoxaparin) has been used as prophylaxis for a month after oncologic surgery, with reduction in VTE. ${ }^{21}$ Our results suggest that a similar regimen for patients undergoing pneumonectomy might be warranted. Prophylaxis to 1 month seems to be an appropriate interval because the majority of VTE cases are clustered in this period.

The only risk factor we identified for VTE after pneumonectomy was increased pack-years of smoking. Smoking increases cardiovascular and cancer risk, but the reason for its association with VTE is unclear, except in its combined use with oral contraceptives. ${ }^{22}$

Death after VTE and its risk factors. Patients who undergo pneumonectomy for malignancy are at high risk of VTE despite prophylaxis and of dying directly from VTE or other causes. We found that mortality of patients with VTE was high, both in those who had pulmonary embolism and in those who had deep vein thrombosis alone.

We found pulmonary embolism after pneumonectomy to be poorly tolerated. Pneumonectomy alone causes increased right ventricular afterload; $50 \%$ of patients given diagnoses of pulmonary embolism have echocardiographic findings of right ventricular dysfunction and increased pulmonary pressures. ${ }^{23,24}$ The added strain pulmonary embolism places on 
a right ventricle already attempting to compensate for increased afterload might explain the high mortality we observed. This theory is supported by the fact that the only risk factor for death after VTE that we identified was reduced pulmonary function.

Comparison of outcome. Patients with VTE had substantially worse outcomes than expected. Studies of VTE in patients with cancer have shown that they have a 4- to 8 -fold higher risk of dying than if this complication had not occurred. $^{2,25-27}$ This was attributed both to the mortality associated with VTE and to its being a marker of more aggressive malignancies, and it was true for patients with locoregional, as well as metastatic, disease. Patients with a higher disease burden might be more likely to have VTE. ${ }^{28}$ In fact, patients who undergo pneumonectomy tend to have more central and larger tumors. Our study shows that VTE is an independent marker for decreased survival for thoracic malignancies.

\section{Limitations}

The main limitation of our study is that although we have identified risk factors for VTE (eg, smoking) and shown that poor pulmonary function is a marker of poor outcome, these are not modifiable when patients present for surgical intervention. Furthermore, although it is intuitive that pulmonary embolism portends decreased survival, it is unclear how deep vein thrombosis alone contributes to higher mortality; pathophysiology of this remains to be explained.

We have likely underestimated the true prevalence of VTE because we obtained imaging studies only on patients with clinical signs of VTE. In addition, we did not perform preoperative screening for deep vein thrombosis or pulmonary embolism, although 6 patients in our series were given preoperative diagnoses of VTE, 4 with deep vein thrombosis and 2 with pulmonary embolism. It is well known that patient history and physical examination are of limited value in diagnosing deep vein thrombosis. ${ }^{29}$ Screening for VTE is an important consideration, although routine surveillance with noninvasive tests has low sensitivity and specificity. ${ }^{30}$ The lack of preoperative and postoperative screening for VTE in our study might have caused us to underestimate its prevalence and overestimate its medical consequences. Asymptomatic patients with VTE might have had benign clinical courses. Finally, this study does not explain whether VTE after pneumonectomy is a direct cause of poor outcome or only a marker of it.

\section{Conclusions}

Our study has shown that VTE is a common event after pneumonectomy and that current prevention and treatment efforts are inadequate. Preoperative and postoperative screening for VTE might be warranted in this high-risk population. Preoperative screening might include duplex scanning, as well as screening hemostatic factors for hypercoagulable state. Postoperative screening would be duplex scanning on postoperative day 7 before discharge. Although this study focused specifically on patients undergoing pneumonectomy, the conclusions are likely applicable to all patients undergoing thoracotomy for malignancy because of the similar risk factors, surgical procedure, and perioperative course. Future studies should also be directed at decreasing the occurrence of VTE through an improved prophylaxis regimen and then evaluating whether this enhances outcome.

\section{References}

1. Clagett GP, Reisch JS. Prevention of venous thromboembolism in general surgical patients. Results of meta-analysis. Ann Surg. 1988; 208:227-40.

2. Blom JW, Osanto S, Rosendaal FR. The risk of a venous thrombotic event in lung cancer patients: higher risk for adenocarcinoma than squamous cell carcinoma. J Thromb Haemost. 2004;2:1760-5.

3. Boyle CA, Decoufle P. National sources of vital status information: extent of coverage and possible selectivity in reporting. Am J Epidemiol. 1990;131:160-8.

4. Newman TB, Brown AN. Use of commercial record linkage software and vital statistics to identify patient deaths. J Am Med Inform Assoc. 1997;4:233-7.

5. Blackstone EH, Naftel DC, Turner ME Jr. The decomposition of time-varying hazard into phases, each incorporating a separate stream of concomitant information. J Am Stat Assoc. 1986;81:615-24.

6. Breiman L. Bagging predictors. Machine Learning. 1996;24:123-40.

7. Blackstone EH. Breaking down barriers: helpful breakthrough statistical methods you need to understand better. J Thorac Cardiovasc Surg. 2001;122:430-9.

8. David HA, Moeschberger ML. The theory of competing risks. New York: Macmillan; 1978.

9. Sergeant P, Blackstone E, Meyns B. Can the outcome of coronary bypass grafting be predicted reliably? Eur J Cardiothorac Surg. 1997; 11:2-9.

10. Kalweit G, Huwer H, Volkmer I, Petzold T, Gams E. Pulmonary embolism: a frequent cause of acute fatality after lung resection. Eur J Cardiothorac Surg. 1996;10:242-7.

11. Kommareddy A, Zaroukian MH, Hassouna HI. Upper extremity deep venous thrombosis. Semin Thromb Hemost. 2002;28:89-99.

12. Bona RD. Thrombotic complications of central venous catheters in cancer patients. Semin Thromb Hemost. 1999;25:147-55.

13. Couban S, Goodyear M, Burnell M, Dolan S, Wasi P, Barnes D, et al. Randomized placebo-controlled study of low-dose warfarin for the prevention of central venous catheter-associated thrombosis in patients with cancer. J Clin Oncol. 2005;23:4063-9.

14. Walshe LJ, Malak SF, Eagan J, Sepkowitz KA. Complication rates among cancer patients with peripherally inserted central catheters. J Clin Oncol. 2002;20:3276-81.

15. Dalla-Volta S, Palla A, Santolicandro A, Giuntini C, Pengo V, Visioli $\mathrm{O}$, et al. PAIMS 2: alteplase combined with heparin versus heparin in the treatment of acute pulmonary embolism. Plasminogen activator Italian multicenter study 2. J Am Coll Cardiol. 1992;20:520-6.

16. Ziomek S, Read RC, Tobler HG, Harrell JE Jr, Gocio JC, Fink LM, et al. Thromboembolism in patients undergoing thoracotomy. Ann Thorac Surg. 1993;56:223-7.

17. Sugarbaker DJ, Jaklitsch MT, Bueno R, Richards W, Lukanich J, Mentzer SJ, et al. Prevention, early detection, and management of complications after 328 consecutive extrapleural pneumonectomies. J Thorac Cardiovasc Surg. 2004;128:138-46.

18. Huber O, Bounameaux H, Borst F, Rohner A. Postoperative pulmonary embolism after hospital discharge. An underestimated risk. Arch Surg. 1992;127:310-3.

19. Geerts WH, Pineo GF, Heit JA, Bergqvist D, Lassen MR, Colwell $\mathrm{CW}$, et al. Prevention of venous thromboembolism: the Seventh ACCP 
Conference on Antithrombotic and Thrombolytic Therapy. Chest. 2004; 126(suppl):338S-400S.

20. Geerts WH, Jay RM, Code KI, Chen E, Szalai JP, Saibil EA, et al. A comparison of low-dose heparin with low-molecular-weight heparin as prophylaxis against venous thromboembolism after major trauma. N Engl J Med. 1996;335:701-7.

21. Bergqvist D, Agnelli G, Cohen AT, Eldor A, Nilsson PE, Le MoigneAmrani A, et al. Duration of prophylaxis against venous thromboembolism with enoxaparin after surgery for cancer. $N$ Engl J Med. 2002;346:975-80

22. Farley TM, Meirik O, Chang CL, Poulter NR. Combined oral contraceptives, smoking, and cardiovascular risk. J Epidemiol Community Health. 1998;52:775-85.

23. Grifoni S, Olivotto I, Cecchini P, Pieralli F, Camaiti A, Santoro G, et al. Short-term clinical outcome of patients with acute pulmonary embolism, normal blood pressure, and echocardiographic right ventricular dysfunction. Circulation. 2000;101:2817-22.

24. Ribeiro A, Lindmarker P, Johnsson H, Juhlin-Dannfelt A, Jorfeldt L. Pulmonary embolism: one-year follow-up with echocardiography Doppler and five-year survival analysis. Circulation. 1999;99:1325-30.

25. Prandoni P, Lensing AW, Cogo A, Cuppini S, Villalta S, Carta M, et al. The long-term clinical course of acute deep venous thrombosis. Ann Intern Med. 1996;125:1-7.

26. Carson JL, Kelley MA, Duff A, Weg JG, Fulkerson WJ, Palevsky HI, et al. The clinical course of pulmonary embolism. $N$ Engl J Med. 1992;326:1240-5.

27. Sorensen HT, Mellemkjaer L, Olsen JH, Baron JA. Prognosis of cancers associated with venous thromboembolism. N Engl J Med. 2000; $343: 1846-50$
28. Lee AY, Levine MN. Venous thromboembolism and cancer: risks and outcomes. Circulation. 2003;107:I17-21.

29. Oudega R, Moons KG, Hoes AW. Limited value of patient history and physical examination in diagnosing deep vein thrombosis in primary care. Fam Pract. 2005;22:86-91.

30. Kearon C, Julian JA, Newman TE, Ginsberg JS. Noninvasive diagnosis of deep venous thrombosis. McMaster Diagnostic Imaging Practice Guidelines Initiative. Ann Intern Med. 1998;128:663-77.

\section{Appendix 1. Variables used in analyses}

Demography

Sex, age at the time of the operation (years), height (centimeters), weight (kilograms)

Cancer variables

Pathologic T stage, tumor size, radiation therapy, chemotherapy

Pulmonary function

Forced expiratory volume in 1 second, forced expiratory volume in 1 second (percentage of normal value), forced vital capacity, forced vital capacity (percentage of normal value)

Cardiac comorbidity

Coronary artery disease, atrial fibrillation, cerebrovascular accident, heart failure

Noncardiac comorbidity

History of diabetes, history of smoking, history of hypertension Experience

Date of operation 